POS PROCEEDINGS

\title{
JEWEL - a Monte Carlo Model for Jet Quenching
}

\author{
Korinna Zapp* \\ Physikalisches Institut, Universität Heidelberg, Philosophenweg 12, D-69120 Heidelberg, \\ Germany
}

ExtreMe Matter Institute EMMI, GSI Helmholtzzentrum für Schwerionenforschung GmbH, Planckstraße 1, 64291 Darmstadt, Germany

E-mail: zapp@physi.uni-heidelberg.de

\section{Johanna Stachel}

Physikalisches Institut, Universität Heidelberg, Philosophenweg 12, D-69120 Heidelberg, Germany

E-mail: stachelephysi.uni-heidelberg.de

\section{Urs Achim Wiedemann}

Physics Department, Theory Unit, CERN, CH-1211 Genève 23, Switzerland

E-mail: Urs.Wiedemann@cern.ch

The Monte Carlo model JewEL 1.0 (Jet Evolution With Energy Loss) simulates parton shower evolution in the presence of a dense QCD medium. In its current form medium interactions are modelled as elastic scattering based on perturbative matrix elements and a simple prescription for medium induced gluon radiation. The parton shower is interfaced with a hadronisation model. In the absence of medium effects JEWEL is shown to reproduce jet measurements at LEP. The collisional energy loss is consistent with analytic calculations, but with JEWEL we can go a step further and characterise also jet-induced modifications of the medium. Elastic and inelastic medium interactions are shown to lead to distinctive modifications of the jet fragmentation pattern, which should allow to experimentally distinguish between collisional and radiative energy loss mechanisms. In these proceedings the main JEWEL results are summarised and a Monte Carlo algorithm is outlined that allows to include the Landau-Pomerantschuk-Migdal effect in probabilistic frameworks.

4th international workshop High-pT physics at LHC 09

February 4-7, 2009

Prague, Czech Republic

* Speaker. 


\section{Introduction}

In ultra-relativistic heavy ion collisions, the produced QCD matter reduces significantly the energy of high transverse momentum partons. While most of the experimental evidence comes from studying the leading hadronic fragments of the parent partons via single inclusive hadron spectra and jet-like particle correlations at RHIC, there is ample motivation for studying mediummodified jets beyond their leading fragments, in particular: i) At the LHC a larger fraction of the entire medium-modified jet fragmentation pattern will become accessible above background. ii) Studying the distribution of subleading fragments is likely to discriminate between different microscopic mechanisms conjectured to underly jet quenching, thereby helping to characterise more precisely the properties of matter tested by jet quenching. iii) Modelling the distribution of subleading jet fragments is essential for any operational procedure aiming at disentangling jets from background or characterising the jet-induced modification of the background. Such reasons motivate the development of tools which account dynamically for the interaction between jet and medium, and which model medium-modified jets on the level of multi-particle final states.

The Monte Carlo technique provides a powerful tool for the simulation of multi-particle final states. In the presence of medium effects, we expect that a parton shower can contribute to understanding 'jet quenching', in particular for the following reasons: i) It reproduces the unmodified jet evolution as vacuum baseline. ii) Energy and momentum can be conserved exactly at each vertex. iii) Different microscopic mechanisms for the interaction between projectile and target can be tested. iv) The modelling of realistic multi-hadron final states is clearly beneficial for comparing theory and data.

In developing JewEL (Jet Evolution With Energy Loss), our main focus was to arrive at a code which allows to study in detail the dynamics relating the evolution of the parton shower to the microscopic modelling of the medium interactions.

This paper aims at summarising the most important features and results of JEWEL 1.0. For more details and more elaborate discussion we refer to $[1,2]$.

\section{Vacuum Baseline}

In the absence of medium effects the JEWEL parton shower is closely related to the massordered shower in PYтнiA 6.4 [3]. It generates fragmentation patterns of a parton with energy $E$, produced in a hard scattering, into a multi-parton final state by probabilistic iteration of the $1 \rightarrow 2$ splitting processes. The virtuality $Q^{2}$ is used as ordering variable, since it is directly related to the parton lifetime. The probability that no splitting occurs between an initial and final virtuality $Q_{\mathrm{i}}^{2}$ and $Q_{\mathrm{f}}^{2}$, respectively, is described by the Sudakov form factor

$$
S_{\mathrm{a}}\left(Q_{\mathrm{i}}^{2}, Q_{\mathrm{f}}^{2}\right)=\exp \left[-\int_{Q_{\mathrm{f}}^{2}}^{Q_{\mathrm{i}}^{2}} \frac{\mathrm{d} Q^{\prime 2}}{Q^{\prime 2}} \int_{z_{-}\left(Q^{\prime 2}, E\right)}^{z_{+}\left(Q^{\prime 2}, E\right)} \mathrm{d} z \frac{\alpha_{\mathrm{s}}}{2 \pi} \sum_{\mathrm{b}, c} \hat{P}_{\mathrm{a} \rightarrow \mathrm{b} c}(z)\right] .
$$

Here, $\hat{P}_{\mathrm{a} \rightarrow \mathrm{bc}}(z)$ are the standard LO parton splitting functions for quarks and gluons $(a, b, c \in\{q, g\})$ and $z$ is the energy fraction carried by the first daughter. The probability density for a splitting to 


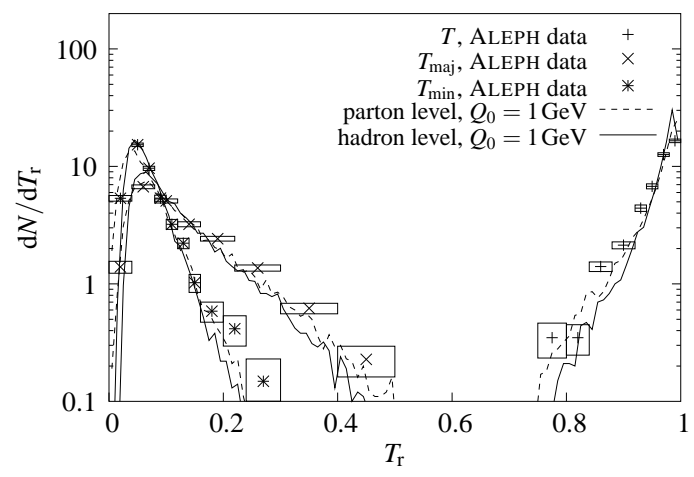

Figure 1: The thrust, thrust major and thrust minor $\left(T_{\mathrm{r}}=\left(T, T_{\mathrm{maj}}, T_{\mathrm{min}}\right)\right)$ distributions for $\sqrt{s}=200 \mathrm{GeV}$ $e^{+} e^{-} \rightarrow q \bar{q} \rightarrow X$ collisions. Data of the ALEPH Collaboration [5] are compared to simulations of JEWEL. Dashed line: parton level after parton shower evolved down to $Q_{0}=1 \mathrm{GeV}$; solid line: hadron level after parton shower evolution followed by hadronisation $\left(Q_{0}=1 \mathrm{GeV}\right)$. Figure taken from [1].

occur at $Q^{2}$ is given by

$$
\Sigma_{\mathrm{a}}\left(Q_{\mathrm{i}}^{2}, Q^{2}\right)=\frac{\mathrm{d} S_{\mathrm{a}}\left(Q_{\mathrm{i}}^{2}, Q^{2}\right)}{\mathrm{d}\left(\ln Q^{2}\right)}=S_{\mathrm{a}}\left(Q_{\mathrm{i}}^{2}, Q^{2}\right) \sum_{\mathrm{b}, c} W_{\mathrm{a} \rightarrow \mathrm{b} c}\left(Q^{2}\right),
$$

where

$$
W_{\mathrm{a} \rightarrow \mathrm{b} c}\left(Q^{2}\right)=\int_{z_{-}\left(Q^{2}, E\right)}^{z_{+}\left(Q^{2}, E\right)} \mathrm{d} z \frac{\alpha_{\mathrm{s}}\left(z(1-z) Q^{2}\right)}{2 \pi} \hat{P}_{\mathrm{a} \rightarrow \mathrm{b} c}(z) .
$$

One thus has to choose the parent parton's virtuality according to (2.2). The type of splitting is selected consistent with $W_{\mathrm{a} \rightarrow \mathrm{bc}}$ and the energy sharing is given by the splitting function $\hat{P}_{\mathrm{a} \rightarrow \mathrm{bc}}(z)$. This procedure is repeated for the daughters until no partons above the infrared cut-off scale $Q_{0}^{2}$ are left. Angular ordering is enforced by allowing only for splittings with decreasing emission angle.

At $Q_{0}$ the parton shower is interfaced with a hadronisation model, that has to be flexible enough to be usable for the complex parton state in heavy-ion collisions. In JEWEL a variant of the Lund string fragmentation [4] is used, where the knowledge about the colour flow is replaced by the assumption of maximal colour correlation of partons close in momentum space.

The JEWEL parton shower is validated against a set of benchmark measurements in $\sqrt{s}=$ $200 \mathrm{GeV} e^{+} e^{-}$collisions at LEP. In Fig. 1 we compare the results of the simulation for the event shape variables thrust $T$, thrust major $T_{\text {maj }}$ and thrust minor $T_{\min }$ to data, with the definitions,

$$
T \equiv \max _{\vec{n}_{T}} \frac{\sum_{i}\left|\vec{p}_{i} \cdot \vec{n}_{T}\right|}{\sum_{i}\left|\vec{p}_{i}\right|} . \quad T_{\text {maj }} \equiv \max _{\vec{n}_{T} \cdot \vec{n}=0} \frac{\sum_{i}\left|\vec{p}_{i} \cdot \vec{n}\right|}{\sum_{i}\left|\vec{p}_{i}\right|} \quad T_{\min } \equiv \frac{\sum_{i}\left|\vec{p}_{i x}\right|}{\sum_{i}\left|\vec{p}_{i}\right|}
$$

Thrust is a measure for how pencil-like an event is. For events, in which all momenta are (anti)parallel to the thrust axis $\vec{n}_{T}, T=1$ and for spherical events $T=1 / 2$. Repeating the analysis in the plane transverse to the thrust axis one obtains thrust major. Thrust minor sums up the components $\vec{p}_{i x}$ of the final particle momenta $\vec{p}_{i}$, which are orthogonal to the plane defined by $\vec{n}$ and $\vec{n}_{T}$.

As seen in Fig. 1, the final state parton shower provides a reasonable description of these jet event shapes over most of the measured range. The JEWEL parton shower is not matched to exact 


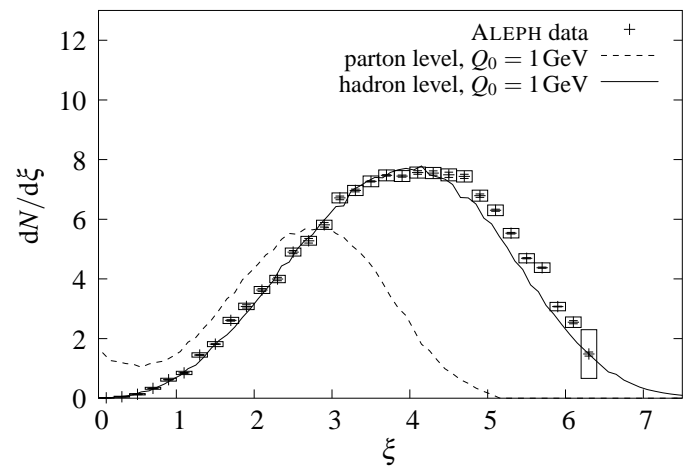

Figure 2: The inclusive distribution $\mathrm{d} N_{\mathrm{ch}} / \mathrm{d} \xi$ with $\xi=\ln \left[E_{\text {jet }} / p_{\text {hadron }}\right]$ of charged hadrons in $e^{+} e^{-} \rightarrow q \bar{q} \rightarrow$ $X$ events at $\sqrt{s}=200 \mathrm{GeV}$. Data of the ALEPH Collaboration [5] are compared to simulations of JEWEL. Dashed line: parton level after parton shower evolved down to $Q_{0}=1 \mathrm{GeV}$; solid line: hadron level after parton shower evolution to $Q_{0}=1 \mathrm{GeV}$ followed by hadronisation. Figure taken from [1].

matrix elements, which may be the reason why it gives too few events with large $1-T, T_{\text {maj }}$ and $T_{\min }$. Fig. 1 also shows that the thrust variables, since they are infrared and collinear safe quantities, are not very sensitive to hadronisation.

The comparison to other event shape variables is of similar or better quality [2], which shows that JEWEL accounts with a sufficient accuracy for global features of the momentum flow.

In comparison to jet event shapes, there are measurements which are more sensitive to the discrete and stochastic nature of the partonic processes underlying the QCD jet fragmentation. One such measurement is the $n$-jet rate, which is based on $k_{\perp}$-clustering algorithms and quantifies the sub-structure of jets. The overall agreement of the simulation results with data is reasonably good, although the 3 -jet rate is somewhat underestimated (possibly due to the missing matrix element matching).

In contrast to the measurements discussed so far, the modelling of single inclusive intra-jet hadron distributions and multi-hadron correlations requires detailed knowledge about the hadronisation mechanism. This is seen for instance in Fig. 2, where we compare results of our simulation to data for the inclusive distribution $\mathrm{d} N_{\mathrm{ch}} / \mathrm{d} \xi$, with $\xi=\ln \left(E_{\text {jet }} / p_{\text {hadron }}\right)$. Irrespective of the choice of $Q_{0}$ the partonic distribution is very hard while hadronisation is expected to soften the distribution considerably. As seen in Fig. 2, JEWEL with the modified string fragmentation describes the data to a level better than a few percent.

We note that in the presence of a high-multiplicity environment, novel hadronisation mechanisms may play a role. Within these uncertainties, which are mainly related to the modelling of hadronisation, we have established that JEWEL provides a reliable baseline for the characterisation of jet quenching phenomena.

\section{Medium Modification of Jets}

We regard the medium as a collection of partons acting as scattering centres. For the case of elastic interactions between the jet and the medium, each scattering centre displays to the partonic projectiles an elastic $2 \rightarrow 2$ scattering cross section $\mathrm{d} \sigma / \mathrm{d} t$. For this exploratory study the medium 
is modelled as an ideal gas of quarks and gluons with a constant temperature $T$. The masses of the scattering centres are fixed to $m_{\text {scatt }}=\mu_{\mathrm{D}}(T) / \sqrt{2}$, where $\mu_{\mathrm{D}}(T)$ is the thermal Debye mass.

To specify the spatiotemporal structure of the parton shower, we start from the estimate that the parton shower evolves down to components of virtuality $Q_{f}$ on a time scale $1 / Q_{f}$. For a parton of energy $E$ and mass $Q_{f}$, this will be time-dilated in the rest frame of the medium to about $E / Q_{f}^{2}$. If the parton originated from the branching of some parton of virtuality $Q_{i}$, then the parton of virtuality $Q_{f}$ existed for a duration of approximately

$$
\tau=\frac{E}{Q_{f}^{2}}-\frac{E}{Q_{i}^{2}} .
$$

For the parent parton, which initialised the parton shower, the lifetime is $\tau=\frac{E}{Q_{i}^{2}}$. In the case of a medium of constant density $n$ scatterings occur during this time with the probability

$$
1-S_{\text {noscatt }}(\tau)=1-\exp \left[-\sigma_{\text {elas }} n \tau \beta\right],
$$

where $\beta$ ist the parton's velocity. For the differential elastic scattering cross section, we choose the LO perturbative $t$-channel parton-parton cross section, which gives the dominant contribution. The $s$-channel contribution is negligible at high energies and the $u$-channel process - apart from being suppressed - transfers essentially all the energy to the scattering centre and is thus irrelevant for elastic energy loss (unless one is interested in jet hadro-chemistry). To make contact with the previous studies, we have chosen two different regularisation schemes for the elastic scattering cross section, namely case I

$$
\sigma^{\text {elas }}=\int_{0}^{\left|t_{\max }\right|} d|t| \frac{\pi \alpha_{s}^{2}\left(|t|+\mu_{D}^{2}\right)}{s^{2}} C_{R} \frac{s^{2}+(s-|t|)^{2}}{\left(|t|+\mu_{D}^{2}\right)^{2}},
$$

which is the default, and case II

$$
\sigma^{\text {elas }}=\int_{\mu_{D}^{2}}^{\left|t_{\max }\right|} d|t| \frac{\pi \alpha_{s}^{2}(|t|)}{s^{2}} C_{R} \frac{s^{2}+(s-|t|)^{2}}{|t|^{2}} .
$$

Elastic scattering may occur between subsequent splitting processes and after the last splitting, provided the parton is still inside the medium.

The model introduced here does not yet include a mechanism of radiative energy loss. Since this may be the main source of energy degradation, we have included an option to enhance the vacuum splitting functions by a factor $\left(1+f_{\text {med }}\right)$,

$$
\hat{P}_{a \rightarrow b c}(z) \longrightarrow\left(1+f_{\text {med }}\right) \hat{P}_{a \rightarrow b c}(z)
$$

as long as the splitting occurs in the medium. This prescription has been argued to display characteristics of radiative energy loss [6].

It is assumed that the hadronisation of a jet is unaffected by the medium, i.e. occurs after all splittings and medium interactions. Since it is not clear, how jets in nuclear collisions hadronise, 


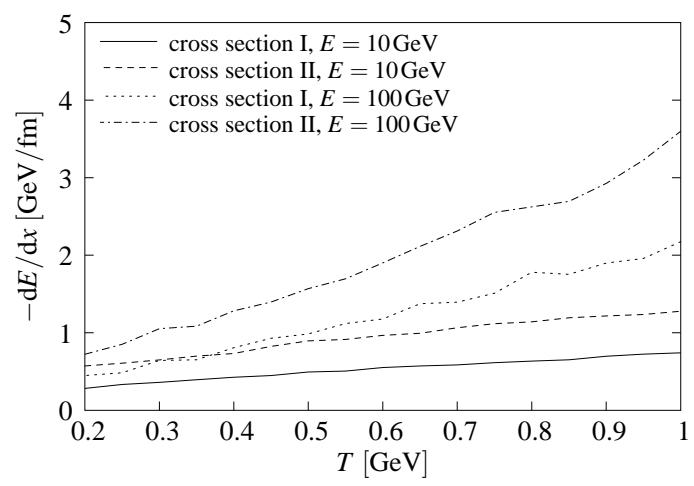

Figure 3: The average parton energy loss $\mathrm{d} E / \mathrm{d} x$ of a quark of energy $E$, undergoing multiple elastic collisions over a path length $L=1 \mathrm{fm}$ in a thermal medium of temperature $T$. Elastic collisions are described by the infrared regulated partonic cross sections case I and case II. Figure taken from [1].

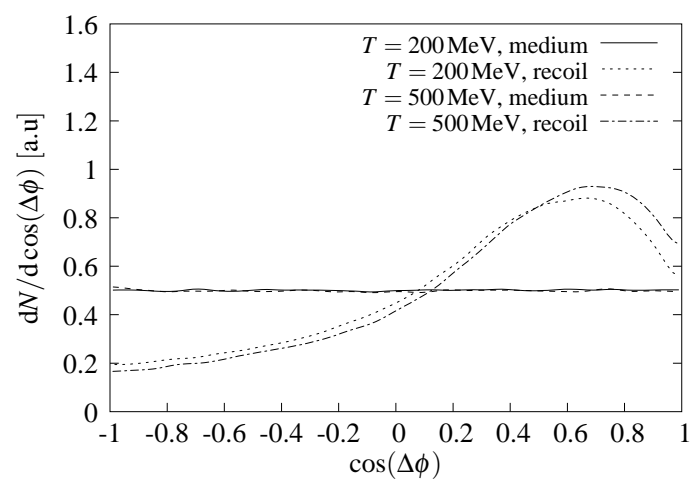

Figure 4: Angle with respect to jet axis of recoiling scattering centres as compared to the undisturbed medium for different temperatures (cross section $\mathrm{I}, E_{\text {jet }}=100 \mathrm{GeV}, L=1 \mathrm{fm}$ ). Hadronisation is not included but may affect these distributions significantly. Figure taken from [1].

we investigate two options. One is that only the hadronisation of the parton shower is considered, whereas in the second the parton shower and the recoiling scattering centres hadronise together.

In Fig. 3, we have calculated the resulting average energy loss for an in-medium path length of $L=1 \mathrm{fm}$ in a medium of temperature $T$. Quantitatively, the models I and II show differences of approximately a factor 2 in $\mathrm{d} E / \mathrm{d} x$ for a $10 \mathrm{GeV}$ parton. Cross section II leads to a larger energy loss, as may be expected since there is minimum momentum transfer. We find that the temperature dependence of $\mathrm{d} E / \mathrm{d} x$ shown in Fig. 3, is consistent with the dependences reported previously $[7-13,15,14]$. The differences between cases I and II are representative of the typical factor 2 uncertainties between different model studies, though some recent studies lead to slightly larger values of $\mathrm{d} E / \mathrm{d} x$ than those shown in Fig. 3, see e.g. [13].

The energy lost by a jet is redistributed amongst the target components. Characterising the recoil of the medium may provide a means to disentangle different mechanisms of parton energy loss. In Fig. 4 the angular distribution of recoiling scattering centres is compared to the undisturbed thermal medium. The recoil moves predominantly in the jet direction with a maximum at $\Delta \phi \sim 0.8$ nearly independent of the temperature. At face value, Fig. 4 indicates that a jet can be accompanied 

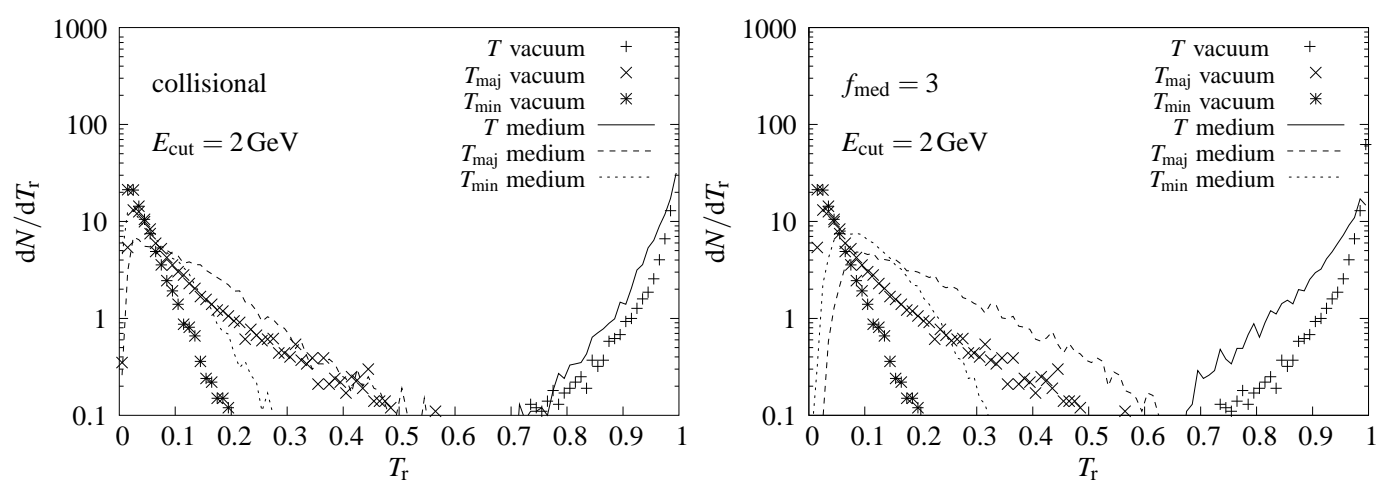

Figure 5: Thrust, thrust major and thrust minor $\left(T_{\mathrm{r}}=\left(T, T_{\mathrm{maj}}, T_{\min }\right)\right)$ for a single $100 \mathrm{GeV}$ jet. The JEWEL parton shower in the vacuum is compared to two scenarios including medium-induced parton energy loss. Left hand side: Collisional energy loss for a medium of $T=500 \mathrm{MeV}$ and in-medium path length $L=5 \mathrm{fm}$ (the recoil is hadronised with the medium). Right hand side: Radiative energy loss for $f_{\text {med }}=3$ and $L=5 \mathrm{fm}$. Only hadrons with energy above $E_{\text {cut }}=2 \mathrm{GeV}$ are included. Figure taken from [1].

by additional multiplicity which has its maximum separated from the jet axis by a characteristic finite angle $\Delta \phi$. We note, however, that whether the partonic distributions of Fig. 4 will or will not change substantially upon hadronisation may depend on details of the hadronisation mechanism.

Next, we study to what extent jet measurements are sensitive to medium effects. Only hadrons with energies above a soft background cut are included in the event shape analyses. This may indicate the extent to which different jet modifications remain visible above the hadronic background in heavy-ion collisions. Fig. 5 shows the thrust distributions for a single jet with and without medium modifications. Collisional energy loss is seen to lead to only a mild broadening of the distributions. The kinematics of elastic $2 \rightarrow 2$ scattering dictates that more energetic projectiles are deflected by smaller angles. The recoiling scattering centres have mostly relatively low momenta so that the medium-induced broadening becomes small if only hadrons of energy above $E_{\text {cut }}=2 \mathrm{GeV}$ are included in the analysis. In contrast to collisional energy loss, single partonic components of medium-induced additional radiation have a higher probability to carry a significant energy fraction of the initial projectile energy. So, on general grounds, one expects that the medium-induced broadening of the distributions in thrust, thrust major and thrust minor will persist even if soft hadrons of energy $E_{\mathrm{h}}<E_{\text {cut }}=2 \mathrm{GeV}$ are dropped from the analysis. This is clearly seen in Fig. 5 (right). The jet rates exhibit the same overall behaviour. Measurements of event shape variables and jet rates may thus provide a tool for disentangling collisional and radiative energy loss.

In Fig. 6, we plot the medium modification of the single inclusive distribution $\mathrm{d} N / \mathrm{d} \xi$ in the presence of collisional and radiative medium effects. If elastic interactions with the medium are included but only the cascade is hadronised, then the total jet multiplicity of projectile hadrons depends only very weakly on the medium, since $2 \rightarrow 2$ processes do not increase the parton multiplicity. However, the total jet multiplicity may increase significantly if recoil partons are counted towards the jet. Fig. 6 also indicates that radiative mechanisms result in a strong increase of intra-jet multiplicities. 


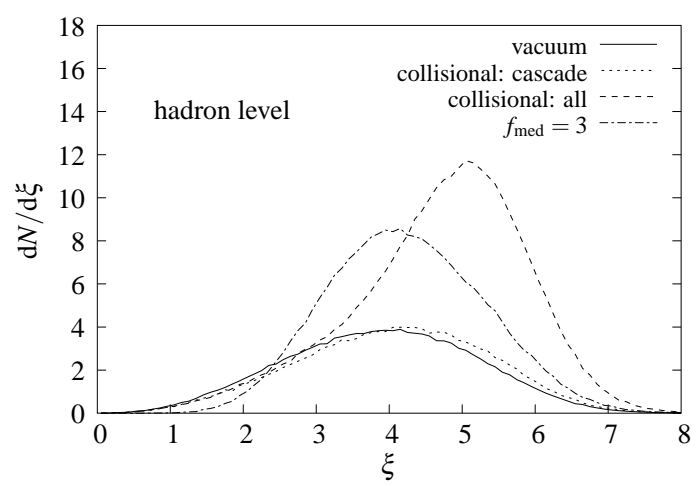

Figure 6: The single inclusive distribution $\mathrm{d} N / \mathrm{d} \xi$ for a single medium-modified quark jet $\left(E_{q}=100 \mathrm{GeV}\right)$ after hadronisation $\left(Q_{0}=1 \mathrm{GeV}\right)$ (only charged hadrons are included). Collisional energy loss is calculated for $T=500 \mathrm{MeV}$ and $L=5 \mathrm{fm}$, with recoil partons either hadronised together with the cascade ('all') or not included in the hadronisation ('cascade'). Medium induced radiation is calculated for $f_{\text {med }}=3$ and $L=5 \mathrm{fm}$. Figure taken from [1].
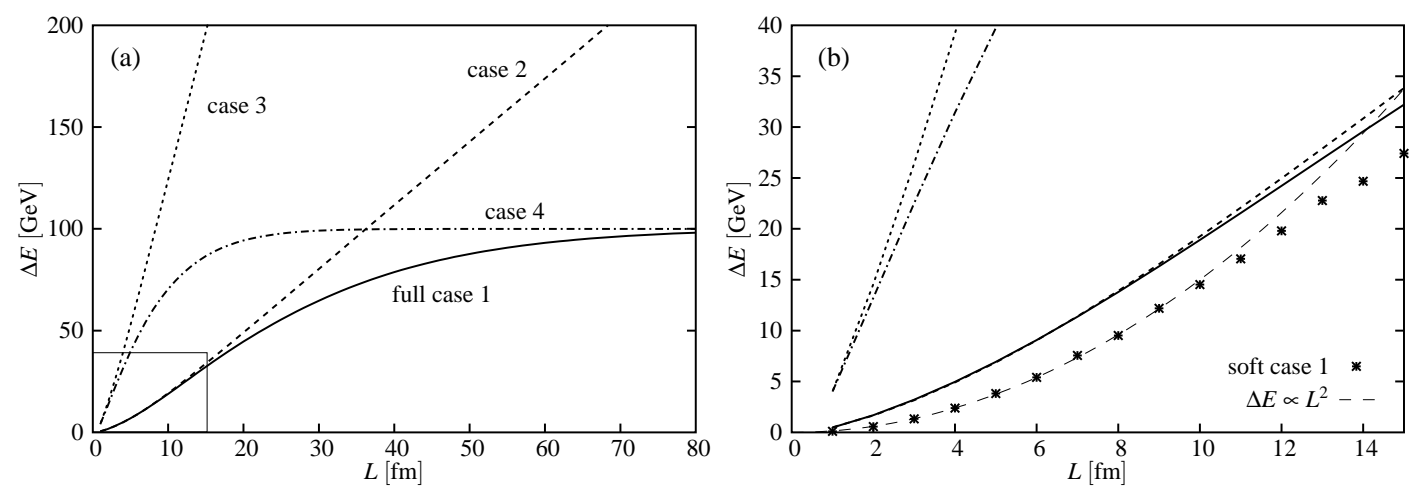

Figure 7: The medium-induced energy loss $\Delta E$ of a quark of initial energy $E_{q}=100 \mathrm{GeV}$ as a function of the in-medium path length $L$. Results for the full MC algorithm with LPM effect and energy conservation (full case 1) are compared to: i) LPM effect without exact energy conservation (case 2), ii) incoherent limit without energy conservation (case 3), iii) incoherent limit with energy conservation (case 4), and iv) full case 1 with constraint that momentum transfer per scattering centre is limited to be soft (soft case 1). The right hand side zooms into the small $L$-region. In this example $L_{\mathrm{c}} \simeq 9 \mathrm{fm}$. Figure taken from [16].

\section{Outlook}

The most important future development of JEWEL is to consistently include inelastic scattering. In [16] a local and probabilistic implementation of the non-abelian Landau-PomerantschukMigdal effect was presented. Analytic calculations [17] suggest that, in general, the LPM effect can be implemented in a probabilistic Monte Carlo algorithm by requiring that the momentum transfer from different scattering centres to the partonic projectile acts totally coherently for gluon production, if it occurs within the formation time $t_{\mathrm{f}}=2 \omega / k_{\perp}^{2}$, and that it acts incoherently, if it occurs after $t_{\mathrm{f}}$. The Monte Carlo implementation of this prescription reproduces as a limiting case the BDMPS [18] results without modifying the gluon emission vertex. But it is more flexible in details of the modelling and has a natural way to implement exact energy-momentum conservation. In Fig. 7 the average energy loss for a projectile quark of initial energy $E_{\mathrm{q}}=100 \mathrm{GeV}$ traversing 
a medium of length $L$ is shown for different cases. Results for the full MC algorithm, including LPM-effect and exact energy-momentum conservation at each vertex are shown as 'full case 1'. Since the BDMPS multiple soft scattering approximation neglects the high- $q_{\perp}$ tail of the scattering cross section, we also consider a case where the momentum transfer is limited to be soft ('soft case 1 '). This case shows the characteristic $L^{2}$-dependence of the average energy loss. Allowing for hard scattering, on the other hand, leads to a loss of coherence and thus increases the energy loss. 'Case 2' is inspired by the high energy approximation assumed in many analytical studies. In this case the gluon energy has to be smaller than the initial quark energy, but the total radiated energy in unconstrained. As expected, this is a good approximation in the region $L<L_{\mathrm{c}} \sim \sqrt{4 \omega_{\max } / \hat{q}}$, but for $L>L_{\mathrm{C}}$ a quasi-incoherent regime is entered, with $\Delta E \propto L$ eventually violating energy conservation. 'Case 3' is a scenario with incoherent gluon emission without energy conservation (but requiring $\omega<E_{\mathrm{q}}$ ), whereas 'case 4' stands for incoherent gluon radiation with energy conservation. Energy conservation can be seen to become the dominant constraint for $L>L_{\mathrm{c}}$. The difference between cases 1 and 4 illustrates the quantitative importance of the LPM-effect.

With this tool a dynamically consistent implementation of inelastic scattering including the LPM-interference in JEWEL seems feasible. Among other planned future extensions are a realistic treatment of the medium and the generalisation to massive quarks.

\section{Acknowledgments}

This research project has been supported via a Marie Curie Early Stage Research Training Fellowship of the European Community's Sixth Framework Programme under contract number (MEST-CT-2005-020238-EUROTHEPHY), by the Helmholtz Alliance Program of the Helmholtz Association, contract HA216/EMMI "Extremes of Density and Temperature: Cosmic Matter in the Laboratory", and the German BMBF.

\section{References}

[1] K. Zapp, G. Ingelman, J. Rathsman, J. Stachel and U. A. Wiedemann, Eur. Phys. J. C 60 (2009) 617 [arXiv:0804.3568 [hep-ph]].

[2] K. Zapp, PhD thesis, Ruprecht-Karls Universität Heidelberg (2008)

[3] T. Sjostrand, S. Mrenna and P. Skands, JHEP 0605 (2006) 026 [arXiv:hep-ph/0603175].

[4] B. Andersson, G. Gustafson, G. Ingelman and T. Sjostrand, Phys. Rept. 97 (1983) 31.

[5] A. Heister et al. [ALEPH Collaboration], Eur. Phys. J. C 35 (2004) 457.

[6] N. Borghini and U. A. Wiedemann, arXiv:hep-ph/0506218.

[7] J. D. Bjorken, preprint Fermilab-pub-82-059-thy.

[8] M. H. Thoma and M. Gyulassy, Nucl. Phys. B 351 (1991) 491.

[9] E. Braaten and M. H. Thoma, Phys. Rev. D 44 (1991) 2625.

[10] M. Djordjevic, Phys. Rev. C 74 (2006) 064907 [arXiv:nucl-th/0603066].

[11] A. Adil, M. Gyulassy, W. A. Horowitz and S. Wicks, Phys. Rev. C 75 (2007) 044906 [arXiv:nucl-th/0606010]. 
[12] B. G. Zakharov, JETP Lett. 86 (2007) 444 [arXiv:0708.0816 [hep-ph]].

[13] S. Peigné and A. Peshier, arXiv:0802.4364 [hep-ph].

[14] K. Zapp, G. Ingelman, J. Rathsman and J. Stachel, Phys. Lett. B 637 (2006) 179 [arXiv:hep-ph/0512300].

[15] S. Domdey, G. Ingelman, H. J. Pirner, J. Rathsman, J. Stachel and K. Zapp, Nucl. Phys. A 808 (2008) 178 [arXiv:0802.3282 [hep-ph]].

[16] K. Zapp, J. Stachel and U. A. Wiedemann, arXiv:0812.3888 [hep-ph].

[17] U. A. Wiedemann, Nucl. Phys. B 588 (2000) 303. [arXiv:hep-ph/0005129].

[18] R. Baier, Y. L. Dokshitzer, A. H. Mueller, S. Peigné and D. Schiff, Nucl. Phys. B 484 (1997) 265. [arXiv:hep-ph/9608322]. 